Monográfico: Reflujo Vesicoureteral

Arch. Esp. Urol., 61, 2 (328-334), 2008

\title{
REFLUJO VESICOURETERAL CONTRALATERAL DESPUES DEL TRATAMIENTO ENDOSCÓPICO DEL REFLUJO UNILATERAL PRIMARIO
}

Manuel García Mérida, Jairo Moreno Román, Carlos Miguélez Lago, Francisca Rius Díazl, Moisés Mieles Cerchar y Eulogio Galiano Duro.

Sección de Urología Pediátrica. Unidad Clínica de Cirugía Pediátrica. Hospital Universitario Materno-Infantil. Hospital Regional Universitario Carlos Haya. Málaga.

Cátedra de Bioestadística'. Departamento de Medicina Preventiva y Salud Pública. Universidad de Málaga. Málaga. España.

Resumen.- OBJETIVO: conocer la incidencia de nuevo RVU contralateral y la evolución del mismo en los niños con reflujo vesicoureteral (RVU) primario unilateral sometidos a tratamiento endoscópico (TE).

MÉTODOS: En un periodo de 7,5 años se han realizado un total de 228 antirreflujos endoscópicos en niños con RVU esencial, de los cuales 90 (39,5\%) han sido unilaterales. Los criterios de inclusión fueron padecer RVU unilateral primario tratado con TE y no haber tenido reflujo contralateral previo. Se valoraron: edad, sexo, número de cistouretrogramas mic cionales seriados (CUMS) previos al procedimiento quirúrgi-
Manuel García Mérida Avda. Juan Sebastián Elcano, 70 - 3을 29017 Málaga. (España). mgarciamerida@gmail.com
Co, grado y lado del RVU inicial y del contralateral, nefropatía ipsi y contralateral, indicaciones de la cirugía inicial, material y volumen utilizado para el TE, y evolución del reflujo. También se realiza una revisión actualizada de la literatura con un metaanálisis para comparar los resultados.

RESULTADOS: Seis niños (6,7\%) presentaron nuevo RVU contralateral. La edad media fue de 3,3 años. El sexo fue masculino en 2 y femenino en 4. Los grados de RVU inicial fueron: II en uno, III en tres y IV en dos. Cuatro pacientes tenían historia de disfunción vesical. El nuevo RVU contralateral fue grado II en 5 niños y grado III en el restante. En cinco niños persistió RVU inicial, siempre de menor grado que el previo, y apareció el nuevo reflujo contralateral; y en uno solo desapareció en el lado inicial y se presentó en el contralateral. Los cinco niños con RVU bilateral se reinyectaron y curaron todos menos uno en que persistió el RVU inicial y está pendiente de reinyección. El niño que presentó solo RVU II contralateral está sometido a observación. En el metanálisis realizado se han encontrado nueve 9 trabajos y tiene una incidencia media de 8,2\%.

CONCLUSIÓN: El RVU Contralateral es una complicación relativamente frecuente en los RVU unilaterales primarios tratados endoscópicamente, pero que no justifica realizar el tratamiento bilateral simultáneo. Éste solo está indicado si existe historia de RVU contralateral previo. La Etiología del RVU Contralateral no esta claramente establecida pero la disfunción vesical puede influir en su aparición.

Palabras clave: Reflujo Vesicoureteral. Reflujo vesicoureteral Contralateral. Tratamiento Endoscópico del Reflujo. Complicaciones del tratamiento del Reflujo vésico ureteral. Metaanálisis 
Summary.- OBJECTIVES: To know the incidence of new contralateral VUR and its evolution in children with primary unilateral vesicoureteral reflux (VUR) managed with endoscopic treatment (ET).

METHODS: During 7.5 years a total of 228 children with primary VUR underwent endoscopic implantation of bulking material, 90 of them (39,5\%) have been unilateral. The inclusion criterion was: unilateral primary VUR managed with ET, without previously contralateral VUR. Collected data included: patient age, gender, indications for surgery, number of preoperative cystourethrograms, preoperative and new contralateral postoperative VUR grades, nephropathy in the ipsilateral or contralateral sides, type and volume of bulking material used, and VUR outcome. An update bibliographic review with methanalysis is also performed to compare results.

RESULTS: Six children $(6,7 \%)$ developed new contralateral VUR. Mean age was 3.3 years. Four patients were females and 2 males. The bulking material used was polydimethylsiloxane in one and Dextranomer/Non animal stabilished hyaluronic acid in 5. The initial grades of primary VUR were: II in 1 case, III in 3, and IV in 2. Four patients had previous history of bladder dysfunction. The new contralateral VUR was II in 5 and III in one. In 5 patients initial VUR persisted, always of lower grade than previously, and new contralateral VUR appeared. In one patient initial VUR disappeared and appeared in the contralateral side. Five patients were reinjected and VUR was cured, except one who is waiting for a new endoscopic procedure. One patient with grade II contralateral VUR is under observation. In the metanalysis performed nine issues have been found with an incidence of $8,2 \%$.

CONCLUSION: Contralateral VUR is a relatively frequent complication in unilateral primary VUR treated by endoscopic procedures (6.7\%), but not enough as to perform bilateral endoscopic treatment in all unilateral VUR. Contralateral VUR etiology is not clear but bladder dysfunction can be an important factor.

Keywords: Vesicoureteral Reflux. Contralateral Vesicoureteral Reflux. Endoscopic treatment. Methanalysis. Endoscopic Treatment Complications.

\section{INTRODUCCIÓN}

Desde hace unos años, el tratamiento endoscópico (TE) del reflujo vesico-ureteral (RVU) es la técnica de corrección del RVU mas utilizada, debido a que es un procedimiento sencillo, mínimamente invasivo, poco traumático para el paciente, que se realiza en hospital de día o en corta estancia y que tiene un alto porcentaje de curación con mínimas complicaciones. Para el TE se han utilizado varios materiales como el teflón, colágeno, condrocitos antólogos, polidimetilsiloxano, etc. En 2001 la Food and Drug Administration de Estados Unidos de América aceptó el dextranómero con ácido hialurónico estabilizado no animal (DX/NASHA) como tratamiento para el RVU y su utilización se ha generalizado. El porcentaje de curación con el TE oscila entre el 68 y el $92 \%$ dependiendo del grado de $R V U$ y de la técnica utilizada $(1,2)$. Con este procedimiento se han descrito muy pocas complicaciones, siendo una de las más frecuentes, dentro de su rareza, el RVU Contralateral (RVUC). Cuando aparece supone una morbilidad añadida puesto que puede ser necesario, según el grado, continuar con profilaxis antibiótica (PA), realizar nuevos estudios radiológicos o someterlo a una nuevo TE.

Se analizan los resultados de nuestra Unidad de Urología Pediátrica y se realiza una revisión de la literatura de esta complicación.

\section{OBJETIVO}

Conocer la incidencia y evolución de RVU Contralateral en los casos de RVU primario sometidos a TE.

\section{MATERIAL Y MÉTODO}

\section{Casuística}

En un período de 7,5 años (marzo de 1998 a diciembre de 2006) se han realizado un total de 228 antirreflujos endoscópicos en niños con RVU primario o esencial, de los cuales $90(39,5 \%)$ han sido unilaterales y son el objetivo del presente estudio.

Los criterios de inclusión fueron: padecer RVU primario unilateral tratado con $T E$, no haber tenido RVU previo, y tener un seguimiento postoperatorio superior a 6 meses.

Se valoraron: edad, sexo, número de cistouretrogramas miccionales seriados (CUMS) previos al procedimiento quirúrgico, grado y lado del reflujo inicial y del contralateral, existencia de nefropatía ipsi y contralateral, indicaciones de la cirugía inicial, material y volumen utilizado para el antirreflujo endoscópico y evolución del nuevo RVUC. El grado de RVU se valoró según el sistema internacional para graduación del reflujo (grados I a V).

La técnica de punción realizada desde 1998 fue el STING descrita por O'Donell y Puri (3) y desde el año 2005 la modificación de Kirsch y cols. (1). Esta modificación del STING es la "Hydrodistension Inyection Technique" (HIT). Con un cistoscopio del 9,5 Fr. con canal de trabajo recto del 5 , se llena parcialmente la vejiga; con ello se facilita la localización de los meatos y la inyección. Se realiza hidrodistensión de los meatos Con aguja metálica de calibre 3,7 Fr. se pincha a las 6 horas en la submucosa del uréter intramural y se introduce la aguja uno o dos milímetros después de terminar el bisel; se inyecta lentamente y sin mover la aguja. Se observa la elevación progresiva de la mucosa vesical hasta formar un buen habón que debe tener la imagen de un volcán con el cráter cerrado en la cima. La 
cantidad de material inyectado varió con el tiempo. Cuando se utilizó polidimetilsiloxano la cantidad habitual fue de $0,5 \mathrm{ml}$ por meato aproximadamente, igual que en los primeros casos de DX/NASHA. Actualmente sólo utilizamos este último material e inyectamos aproximadamente 1 mililitro para cada uréter, dependiendo de la hidrodistensión pre y post punción. Si despues de la inyección persiste la hidrodistensión se reinyecta en uno o mas puntos para conseguir una mejor oclusión del meato. Inicialmente se valoró solo la forma y posición del meato ureteral y desde el 2005, se hace constar además, la hidrodistensión de los mismos.

El procedimiento se realiza con anestesia general y mascarilla laríngea. En la inducción anestésica se administra profilaxis antibiótica con cefalosporina de segunda generación. Puede irse a su domicilio a las 5-6 horas de la cirugía, tras la recuperación anestésica completa y comprobación de micciones normales.

A las cuatro semanas del procedimiento se realiza ecografía para valorar la existencia de una posible dilatación del tracto urinario superior y el habón del material inyectado en la vejiga. A los tres meses se realiza CUMS radiológico para valorar la existencia de RVU ipsi o contralateral.

\section{Metaanálisis}

Se ha realizado una revisión sistemática de la literatura con metanálisis de las series válidas para estudio, para conocer la incidencia, evolución y tratamiento de esta complicación en el antirreflujo endoscópico. Se han utilizado "PubMed" y "Ovid" como buscadores de literatura médica con las palabras clave "vesicoureteral reflux", "endoscopic treatment", "contralateral reflux" y "complication".

\section{RESULTADOS}

La edad media de los 90 casos de RVU esencial unilateral ha sido de 3,6 años (Rango 1 a 12 años). El sexo fue femenino en $49(54,4 \%)$ y masculino en $41(45,6 \%)$. Los grados de RVU inicial fueron: 26 II $(28,9 \%), 42$ III $(46,7 \%), 19$ IV $(21,1 \%)$ y $3 \vee(3.3 \%)$. En 40 niños $(44,4 \%)$ el TE se realizó con polidimetilsiloxano y en $50(55,6 \%)$ con DX/NASHA.

Seis niños $(6,7 \%)$ presentaron nuevo RVU Contralateral en el CUMS de control efectuado a los tres meses del procedimiento. La edad media de los mismos fue de 3,3 años con un rango de 14 meses a 6 años. El sexo fue femenino en $4(66,7 \%)$ y masculino en $2(33,3 \%)$.

\section{Estudio de los 6 casos con RVUC}

Situación inicial. El motivo de diagnóstico del RVU inicial fue infección del tracto urinario en cinco $(83,3 \%)$ y ser hermano de paciente con RVU de alto grado el restante. Los grados de RVU inicial fueron: 1 II $(16,7 \%), 3$ III $(50 \%)$ y 2 IV $(33,3 \%)$. Cinco $(83,3 \%)$ eran del lado izquierdo. Cuatro pacientes $(66,7 \%)$ tenían historia de disfunción vesical: dos presentaban hábito retencionista, uno vejiga hiperactiva y el restante disinergia detrusor-esfínter.

En la gammagrafía renal preoperatoria, con ácido dimercaptosuccinico (DMSA Tc-99) existía nefropatía de reflujo ipsilateral en dos (33.33\%) y ninguno tenía lesiones en el riñón contralateral.

El número de CUMS previos al tratamiento fue: 3 en 2 niños, 2 en 3 niños y 1 en el restante, con una media de 2.16 CUMS preoperatorios / paciente. En ninguno de ellos se observó RVU contralateral.

TABLA I. PACIENTES QUE DESARROLLARON RVU CONTRALATERAL.

\begin{tabular}{|c|c|c|c|c|c|c|}
\hline Caso & o RVU inicial & $\begin{array}{c}\text { Resultado RVU } \\
\text { inicial }\end{array}$ & $\varrho$ RVU Contral & $\begin{array}{c}\text { Tto. RVU inicial } \\
\text { y Contral }\end{array}$ & $\begin{array}{c}\text { Resultado RVU } \\
\text { Contral }\end{array}$ & Otros síntomas \\
\hline 1 & III & I Curado & II & Reinyección & Curado & Retencionista \\
\hline 2 & III & II Curado & II & Reinyección & Curado & Disinergia \\
\hline 3 & IV & III Curado & III & Reinyección & Curado & No continente \\
\hline 4 & II & Curado & II & Reinyección & Curado & Curado \\
\hline 5 & IV & III Persiste & $\|$ & Reinyección & Curado & V. hiperactiva \\
\hline 6 & III & Curado & $\|$ & Observación & Pdte CUMS & \\
\hline
\end{tabular}

RVU: Reflujo Vésico Ureteral. RVU: Grado de reflujo Vésico Ureteral. Contral: Contralateral.

Tto: tratamiento. Pdte: Pendiente. CUMS: Cistouretrografía miccional seriada 


\begin{tabular}{|c|c|c|c|c|c|c|c|c|c|c|c|c|}
\hline 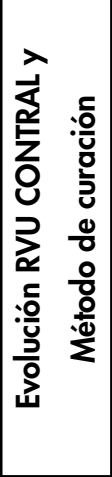 & 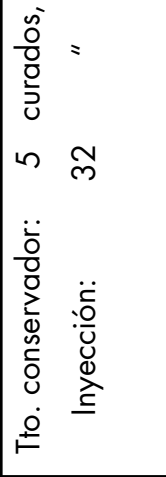 & 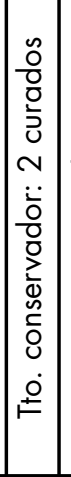 & 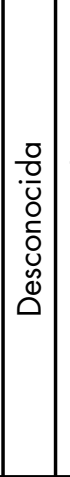 & $\left|\begin{array}{l|}0 \\
\cdot \frac{0}{u} \\
0 \\
0 \\
0 \\
0 \\
0 \\
0 \\
0\end{array}\right|$ & $\left|\begin{array}{l}0 \\
\cdot \frac{0}{u} \\
0 \\
o \\
0 \\
0 \\
0 \\
0 \\
0\end{array}\right|$ & 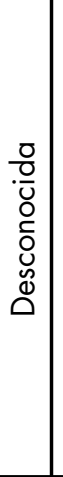 & 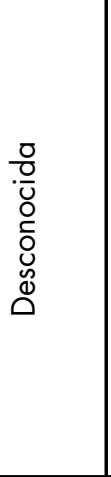 & 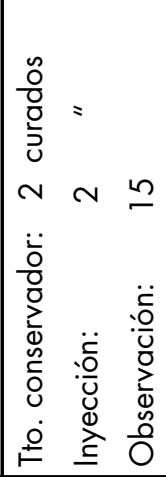 & 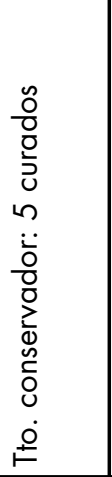 & 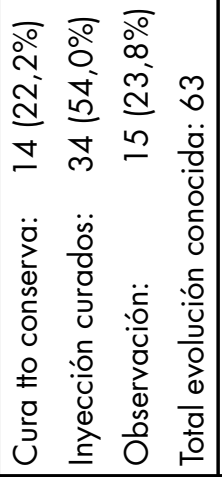 & 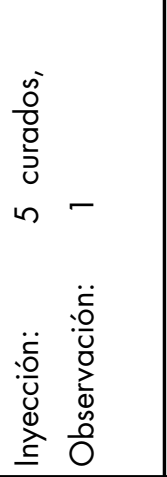 & 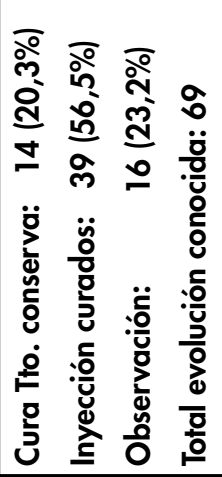 \\
\hline 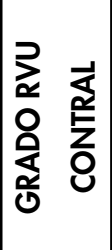 & 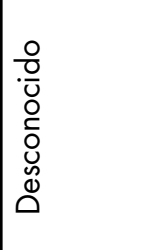 & $=$ & 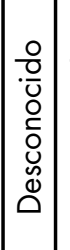 & $\left|\begin{array}{l}0 \\
: \frac{0}{U} \\
0 \\
0 \\
0 \\
0 \\
0 \\
0 \\
0\end{array}\right|$ & 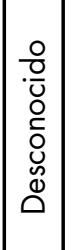 & 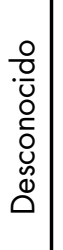 & $\begin{array}{ll}\simeq & 0 \\
\doteq & \ddot{\geq}\end{array}$ & $\begin{array}{lll}\wedge & \infty & \sim \\
\ddot{-} & \ddot{\equiv} & \doteq\end{array}$ & 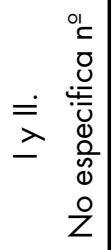 & 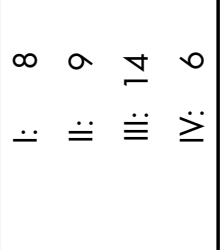 & 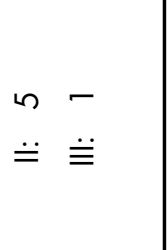 & $\begin{array}{llll}\infty & \Xi & \curvearrowleft \\
\ddot{-} & \ddot{=} & \ddot{\equiv}\end{array}$ \\
\hline 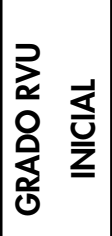 & 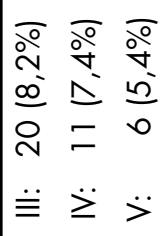 & 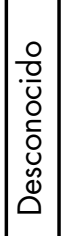 & $\geq$ & 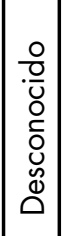 & 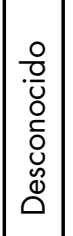 & 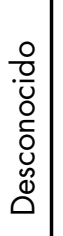 & 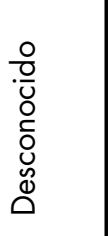 & 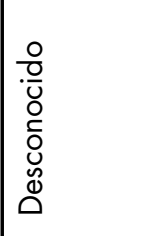 & 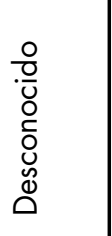 & 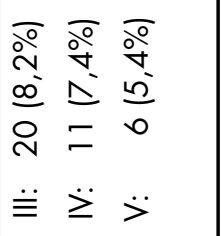 & 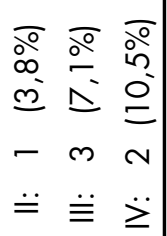 & 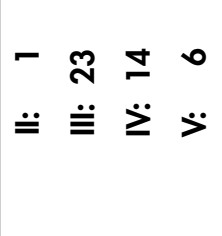 \\
\hline 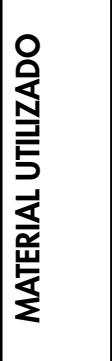 & 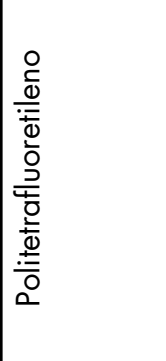 & 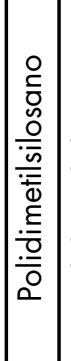 & 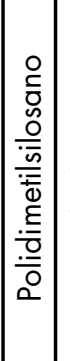 & 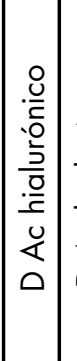 & 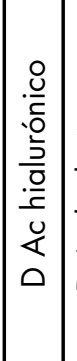 & 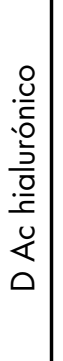 & 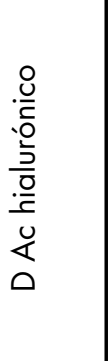 & 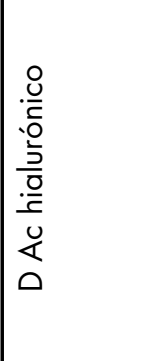 & 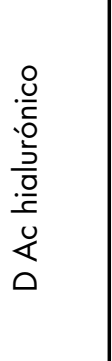 & 1 & 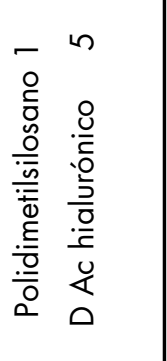 & 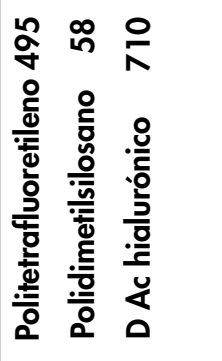 \\
\hline 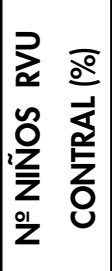 & 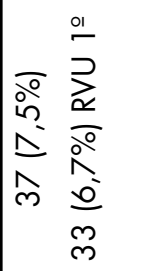 & 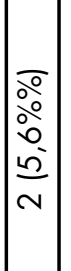 & $\begin{array}{l}\overline{0} \\
\circ \\
\infty \\
\pm \\
- \\
-\end{array}$ & $\mid \begin{array}{c}0 \\
i n \\
i \\
\\
m \\
m\end{array}$ & $\left|\begin{array}{c}\overline{0} \\
\stackrel{0}{0} \\
\infty \\
0 \\
0\end{array}\right|$ & $\frac{\frac{0}{\circ}}{\frac{a}{N}}$ & $\begin{array}{l}\frac{0}{0} \\
\frac{1}{0} \\
0\end{array}$ & 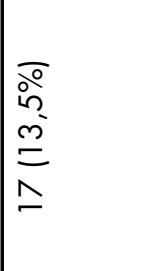 & $\frac{\bar{O}}{\stackrel{\circ}{N}}$ & $\begin{array}{l}\text { वे } \\
\stackrel{\infty}{\infty} \\
\frac{\infty}{a}\end{array}$ & $\begin{array}{l}\frac{0}{0} \\
\frac{0}{0} \\
0\end{array}$ & $\frac{\sqrt{0}}{\frac{\infty}{0}}$ \\
\hline 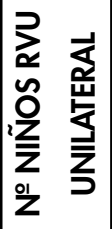 & $\stackrel{2}{\alpha}$ & 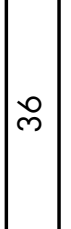 & $\bar{\sim}$ & 文 & 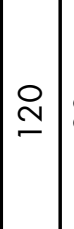 & $\stackrel{\infty}{m}$ & 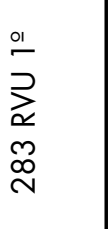 & 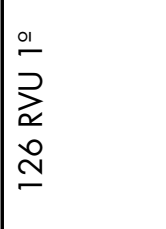 & a & $\underset{\simeq}{\simeq}$ & 음 & ণ్లి \\
\hline 은 & 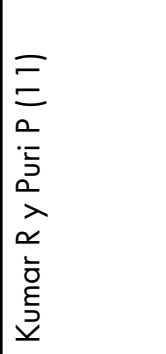 & 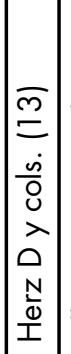 & 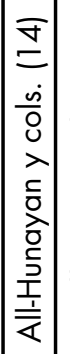 & 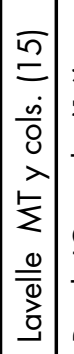 & $\begin{array}{c}0 \\
0 \\
= \\
\dot{0} \\
\dot{0} \\
0 \\
> \\
u \\
\underline{c} \\
\frac{c}{J} \\
0 \\
\alpha\end{array}$ & 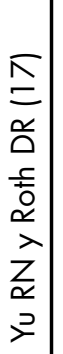 & 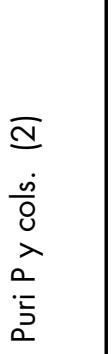 & $\begin{array}{l}\bar{N} \\
= \\
\dot{0} \\
0 \\
0 \\
\lambda \\
\sum \\
0 \\
\frac{0}{0} \\
\frac{E}{W}\end{array}$ & $\begin{array}{l}\infty \\
= \\
\dot{\infty} \\
0 \\
0 \\
\supset \\
0 \\
\frac{3}{2}\end{array}$ & $\begin{array}{l}0 \\
\frac{0}{0} \\
0 \\
0 \\
\frac{0}{0} \\
\frac{0}{0} \\
\frac{0}{0} \\
\frac{0}{0} \\
\frac{0}{0}\end{array}$ & $\begin{array}{l}\frac{8}{0} \\
\frac{0}{0} \\
\sum_{0}^{0} \\
\frac{0}{0} \\
0 \\
\frac{0}{2} \\
0\end{array}$ & 흥 \\
\hline
\end{tabular}


A todos los niños en edad continente se les aconsejó educación y horario miccional. La disinergia se trató con biofeedback, y la hiperactividad vesical con anticolinérgicos.

Tratamiento Endoscópico Inicial. Las indicaciones del tratamiento endoscópico fueron:

- 4 niños por persistencia del RVU despues de 1 año como mínimo de PA.

- 2 niños por existencia de nefropatía.

El TE se realizó con Macroplastique $R^{\circledR}$ en un paciente y con Deflux $\AA^{\circledR}$ en los otros cinco. El volumen medio de material inyectado fue de $0,57 \mathrm{ml}$ con un rango de 0,5 a 1 . El aspecto del meato ureteral contralateral era en herradura en 4, en boca de horno en uno y en hoyo de golf en el restante. La hidrodistensión del meato contralateral solo consta en tres niños y en dos fue positiva, pero no se inyectaron.

Aparición del RVU Contralateral. El CUMS de control a los tres meses del tratamiento endoscópico mostró RVU contralateral grado II en $5(83,3 \%)$ y grado III en 1 (16,7\%).

\section{La evolución del RVU inicial y del nuevo RVU Contralateral se especifica en la Tabla I.}

Grupo A.- En cinco niños (83,3\%) persistió RVU inicial, siempre de menor grado que antes del TE, y apareció el nuevo RVUC. Cuatro niños con persistencia del RVU inicial con un grado II o mayor se reinyectaron bilateralmente y en todos desapareció el contralateral; en uno persiste el RVU en el lado inicial de menor grado que antes del TE (caso 5) y está pendiente de una tercera inyección. Otro paciente (caso 1) con RVU inicial grado III y hábito retencionista, tras la primera inyección disminuyó a grado I pero apareció RVUC grado II que se observó con PA pero hizo una infección urinaria febril por lo que se hizo nuevo TE, con curación bilateral del RVU.

Grupo B.- Sólo en un niño desapareció el RVU inicial y se presentó el RVUC grado II. Está en observación sin profilaxis antibiótica, con horario miccional y lleva seis meses de control sin ninguna incidencia.

\section{DISCUSIÓN}

La complicación más frecuente del TE del RVU es la persistencia del mismo. Otras complicaciones descritas son el RVUC, la obstrucción ureteral y la hematuria franca.

En los casos unilaterales, la aparición de nuevo RVUC, sin historia de haberlo padecido previamente, es una complicación poco frecuente y poco analizada en la literatura.

El RVUC se puede presentar en los niños con RVU unilateral tratados con reimplante ureteral con cirugía abierta y tambien con TE; incluso se ha descrito durante la $\mathrm{PA}$, sin ningún procedimiento quirúrgico.
La etiología del RVUC es desconocida. Se han invocado varios mecanismos para justificarlo, pero ninguno lo explica plenamente. Es probable que el RVUC aparezca por una conjunción de algunos de ellos. Se cree que puede ser debido a:

- Distorsión del trígono durante el procedimiento quirúrgico,

- Existencia de un RVU previo desconocido,

- Historia natural del reflujo,

- Disfunción vesical y/o esfinteriana

- La resolución de un RVU de alto grado aumenta la presión vesical y por el mecanismo de pop-off se desestabiliza en el uréter contralateral (4-7).

RVU Contralateral tras Cirugía Abierta. Se presenta entre el 5.3 y $32 \%$ de todos los procedimientos antirreflujo efectuados $(5,7,8)$. La incidencia varía según la técnica quirúrgica de reimplantación unilateral empleada: se ha descrito 0 a $19 \%$ en Cohen, 9 a $27 \%$ en Leadbetter-Politano, 17\% en Glen-Anderson, 20\% en Paquin, y de 5,3 a 6,8\% en LichGregoire (6-8). Lógicamente el RVUC es menos frecuente en los procedimientos que desestructuran menos el trígono como son los extravesicales $(4,5,8)$. Para evitar esta complicación, si existe historia de RVU contralateral previo, sea del grado que sea, y aunque hubiera desaparecido durante la evolución se recomienda realizar reimplante antirreflujo bilateral.

RVU Contralateral durante la Profilaxis Antibiótica. En 1998, Sparr y cols. (9) describieron que el RVUC se puede presentar en los niños con RVU unilateral sin ningún tipo de cirugía. La incidencia fue del $33 \%$ de los niños con RVU unilateral y en todos los casos el nuevo RVUC fue grado III o menor resolviéndose espontáneamente en el $40 \%$ de los mismos. Un trabajo más reciente manifiesta que cuando el reflujo unilateral es de alto grado existen más posibilidades de desarrollar RVUC y que su resolución espontánea es menos frecuente (10). Por lo tanto el RVUC no está relacionado sólo con la cirugía, si no que puede ser una manifestación de la historia natural del RVU unilateral y del mecanismo del pop-off (9-10).

RVU Contralateral tras Tratamiento Endoscópico. Metaanálisis. En la revisión de la literatura del tratamiento endoscópico del RVU se encontraron un total de 388 citas bibliográficas desde el año 1984 al 2006. Se excluyeron todos los artículos de cirugía abierta y se leyeron todos los resúmenes escritos en revistas de habla inglesa y español. En aquellos en los que en los resultados hablaban de "aparición de nuevo RVUC" se leyó el artículo completo.

Se han encontrado sólo 9 artículos en los que se cita tal complicación del TE inicial, dos son del mismo grupo aunque de años diferentes $(1,11)$, por lo que se hace referencia sólo al trabajo más reciente (Tabla II).

De un total de 1.212 antirreflujos endoscópicos unilaterales, en RVU primarios y secundarios, 99 niños $(8,2 \%)$ presentaron nuevo RVUC, con una frecuencia que oscila entre el 4,8 y el $21 \%(2,11-18)$. Los artículos revisados no ofrecen datos completos del inicio, evolución y tratamiento del RVU contralateral: 3 artículos especifican el 
grado del nuevo RVU contralateral $(2,12,13)$, 2 el grado de RVU inicial de los casos que desarrollaron la complicación $(11,14)$, y solo en 4 se conoce la evolución y tratamiento del mismo $(11-13,18)$.

En nuestra serie el 6,7\% de los niños con RVU unilateral primario desarrolló nuevo RVUC, incidencia algo menor que la media en la literatura $(8,2 \%)$. El grado de RVU inicial fue grado II en un niño, grado III en tres niños y grado IV en dos, lo que representa el $3.8,7.1$ y $10.5 \%$ respectivamente del total de niños de cada grado de RVU. No hubo ninguno del grado $V$ que presentara la complicación. El RVUC parece ser más frecuente con mayor grado de RVU inicial. Este dato parecería sugerir que el mecanismo del pop-off puede ejercer algún papel en el desarrollo del RVUC, aunque Kumar y Puri (1 1), en una amplia serie, especifican que este mecanismo no es la causa del mismo. Dado que ninguna otra serie de tratamiento endoscópico especifica el grado de RVU inicial no se han podido realizar más comparaciones. En nuestra serie las diferencias no son estadísticamente significativas (test Chi cuadrado), posiblemente porque el número de niños es escaso.

En el TE la distorsión del trígono es muy pequeña por lo que este mecanismo parece tener un papel muy escaso en la producción del nuevo RVU. No se han encontrado diferencias significativas entre el volumen del material inyectado a los niños que hicieron la complicación y los que no la tuvieron (12).

El número medio de CUMS previos a la aparición del RVU contralateral fue de 2,2 por niño. En el DMSA ninguno de los riñones que desarrollaron el nuevo RVUC presentaba signos de nefropatía. Estos datos hacen suponer que el nuevo RVUC se ha generado después y puede constituir la historia natural del RVU unilateral.

Estudio de factores influyentes. En nuestra serie el sexo y el aspecto del meato ureteral no parecen ejercer un papel importante en el desarrollo del RVUC. La edad parece un factor determinante pues excepto un paciente de 6 años, todos los demás eran menores de 5 . Un dato que también parece influir es la disfunción vesical ya que la presentaban 4 de los 5 pacientes de nuestra serie en edad continente (80\%) (Tabla I).

Elmore (12) describe un grupo de riesgo, estadísticamente significativo, con altas posibilidades de desarrollar RVUC que son las pacientes de sexo femenino y menores de 5 años; por lo que recomienda que en ellas se realice tratamiento bilateral si la hidrodistensión del meato ureteral es positiva. No obstante, dado el escaso porcentaje de RVUC no se ha encontrado quien recomiende el tratamiento bilateral sistemática en el RVU unilateral.

Evolución y tratamiento. El RVUC suele ser de bajo grado, como ocurrió en nuestra serie, por lo que tiene altas posibilidades de desaparecer espontáneamente con el crecimiento. En la literatura un 22,2\% de los nuevos RVUC en los que se conoce la evolución desapareció sin tratamiento quirúrgico (Tabla II). La resolución o persistencia del RVU inicial influye en el tratamiento. Si éste persiste con un grado mayor de I, se recomienda realizar una segunda inyección en el inicial y una primera en el contralateral, como se hizo en 5 de nuestros pacientes. Si el RVU inicial se ha curado y el contralateral es de bajo grado, como sucedió en otro de niño de esta serie, pensamos que la aptitud inicial debe ser abstencionista, recomendando horario miccional y esperar la evolución. Actualmente en niños mayores de 1 año y con RVU I-II no utilizamos PA. Si los niños con RVUC presentan infecciones urinarias de repetición se recomienda TE. En la revisión de la literatura un $54 \%$ de los casos de RVUC se trató endoscópicamente, auque desconocemos si el RVU inicial estaba curado o no.

\section{CONCLUSIÓN}

En el metanálisis realizado la prevalencia del RVUC en los RVU unilaterales tratados endoscópicamente, ha sido $8,2 \%$ con rango $4.8-21 \%$. En la presente serie la prevalencia en RVUC unilateral primario ha sido $6,7 \%$. Esta baja tasa no justifica realizar el TE bilateral simultáneo en todos los RVU unilaterales primarios. Éste sólo está indicado si existe historia de RVU contralateral previo, aunque hubiera desaparecido espontaneamente.

No obstante parece existir una población de riesgo con más posibilidades de desarrollar RVUC: Pacientes de sexo femenino, menores de 5 años, con hidrodistensión meatal positiva, o historia de disfunción miccional. En estos casos podría estar justificado el TE simultaneo bilateral.

La disfunción vesical y/o uretral puede favorecer la aparición de RVUC.

\section{BIBLIOGRAFÍA Y LECTURAS RECOMENDADAS ("lectura de interés $y^{* *}$ lectura fundamental)}

**1. KIRSCH, A.J.; PEREZ-BRAYFIELD, M.; SMITH, E.A. y cols.: "The modified sting procedure to correct vesicoureteral reflux: Improved results with submucosal implantation within the intramural ureter". J. Urol., 171: 2413, 2004.

2. PURI, P.; PIRKER, M.; MOHANAN, N. y cols.: "Subureteral dextranomer/hialuronic acid injection as first line treatment in management of high grade vesicoureteral reflux". J. Urol., 176: 1856, 2006.

**3. O'DONNELL, B.; PURI, P.: "Treatment of vesicoureteric reflux by endoscopic injection of Teflon". Br. Med. J. (Clin. Res. Ed.), 289: 7, 1984.

**4. ELDER, J.S.: "Guidelines for considerations for surgical repair of vesicoureteral reflux". Curr. Opin. Urol., 10: 579, 2000.

5. MINEVICH, E.; WACKSMAN, J.; LEWIS, A.G. y cols.: "Incidence of contralateral vesicoureteral reflux following unilateral extravesical detrusorrhaphy (ureteroneocystostomy)". 159: 2126, 1998.

6. DIAMOND, D.A.; RABINOWITZ, R.; HOENIG, D.M. y cols.: "The mechanism of new onset contralateral reflux following unilateral ureteroneocystostomy". J. Urol., 156: 665, 1996. 
7. HOENIG, D.M.; DIAMOND, D.A.; RABINOWITZ, R. y cols.: "Contralateral reflux after unilateral ureteral reimplantation". J. Urol., 156: 196, 1996.

8. GARCÍA MÉRIDA, M.; GOSALBEZ, R.; RIUS DÍAZ, F. y cols.: "Reflujo vesicoureteral esencial y reimplantación ureteral extravesical en niños". Actas Urol. Esp., 30: 602, 2006.

9. SPARR, K.E.; BALCOM, A.H.; MESROBIAN, H.G.: "Incidence and natural history of contralateral reflux in patients presenting unilateral disease". J. Urol., 160: 1023, 1998.

**10. BARROSO, U.; CALADO, A.; BARROSO, V. y cols.: "Predictive factors for the development of contralateral reflux in children with unilateral reflux treated conservatively. Abstracts of the ESPU XVIIITH Annual Congress". J. Pediatr. Urol., 3: 17, 2007.

11. KUMAR, R.; PURI, P.: "Newly diagnosed contralateral reflux after successful unilateral endoscopic correction: Is it due to the pop-off mechanism". J. Urol., 158: 1213, 1997.

**12. ELMORE, J.M.; KIRSCH, A.J.; LYLES, R.H. y cols.: "New contralateral vesicoureteral reflux following dextranomer/hialuronic acid implantation of high risk group". J. Urol., 175: 1097, 2006.

13. HERZ, D.; HAFEZ, A.; BAGLI, D. y cols.: "Efficacy of endoscopic subureteral polydimetilsiloxane injection for treatment of vesicoureteral reflux in children: A North American clinical report". J. Urol., 166: 1880, 2001.

14. AL-HUNAYAN, A.A.; KEHINDE, E.O.; ELSALAM, M.A. y cols.: "Outcome of endoscopic treatment for vesicoureteral reflux in children using polydimethilsiloxano". J. Urol., 168: 2181, 2002.

15. LAVELLE, M.T.; CONLIN, M.J.; SKOOG, S.J.: "Subureteral injection of deflux for correction of reflux: Analysis of factor predicting success". Urology, 65: 564,2005

16. ROUTH, J.C.; VANDERSTEEN, D.R.; PFEFFERLE, H. y cols.: "Single center experience with endoscopic management of vesicoureteral reflux in children". J. Urol., 175: 1889, 2006.

17. YU, R.N.; ROTH, D.R.: "Treatment of vesicoureteral reflux using endoscopic injection of nonanimal stabilized hialuronic acid/dextranomer gel: Initial experience in pediatric patients by a single surgeon". Pediatrics, 18: 698, 2006.

18. ALTUG, U.; CAKAN, M.; YILMAZ, S. y cols.: "Are there predictive factors for the outcome of endoscopic treatment of grade III-IV vesicoureteral reflux with dextranómero/hyaluronic acid in children". Pediatr. Surg. Int., 23: 585, 2007. 